Acta Crystallographica Section E

Structure Reports

Online

ISSN 1600-5368

\section{6,6'-Diethoxy-2,2'-[hexane-1,6-diylbis- (nitrilomethanylylidene)]diphenol}

\section{Kwang Ha}

School of Applied Chemical Engineering, The Research Institute of Catalysis, Chonnam National University, Gwangju 500-757, Republic of Korea

Correspondence e-mail: hakwang@chonnam.ac.kr

Received 4 March 2011; accepted 11 March 2011

Key indicators: single-crystal X-ray study; $T=200 \mathrm{~K}$; mean $\sigma(\mathrm{C}-\mathrm{C})=0.004 \AA$; $R$ factor $=0.062 ; w R$ factor $=0.168 ;$ data-to-parameter ratio $=15.5$.

The title compound, $\mathrm{C}_{24} \mathrm{H}_{32} \mathrm{~N}_{2} \mathrm{O}_{4}$, is a polydentate Schiff base and reveals strong intramolecular $\mathrm{O}-\mathrm{H} \cdots \mathrm{N}$ hydrogen bonding between the hydroxy $\mathrm{O}$ atom and the imino $\mathrm{N}$ atom, with an $\mathrm{O} \cdots \mathrm{N}$ distance of 2.570 (3) $\AA$. In the crystal, a centre of inversion is located at the mid-point of the compound. The diiminohexylene chain is almost ideally in the anti conformation, with an average dihedral angle of $179.0^{\circ}$.

\section{Related literature}

For related structures, see: Bermejo et al. (2007); Fun et al. (2009); Ha (2010).

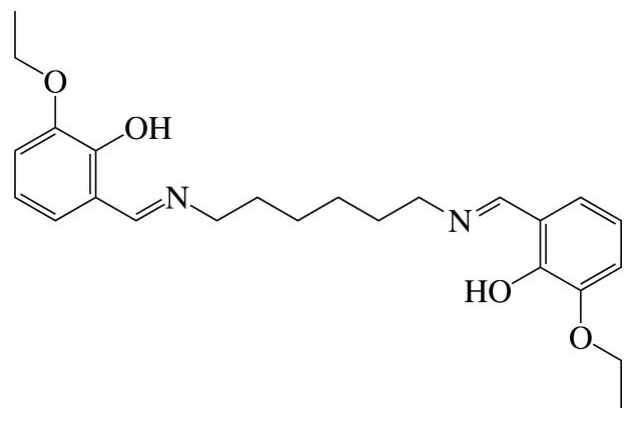

\section{Experimental}

Crystal data

$\mathrm{C}_{24} \mathrm{H}_{32} \mathrm{~N}_{2} \mathrm{O}_{4}$

$M_{r}=412.52$
Triclinic, $P \overline{1}$ $a=6.9094(13) \AA$ $b=6.9184(13) \AA$

$c=11.936(2) \AA$

$\alpha=91.271(5)^{\circ}$

$\beta=99.677$ (4)

$\gamma=102.550(4)^{\circ}$

$V=547.97(18) \AA^{3}$

Data collection

Bruker SMART 1000 CCD diffractometer

Absorption correction: multi-scan (SADABS; Bruker, 2000)

$T_{\min }=0.862, T_{\max }=1.000$

Refinement

$R\left[F^{2}>2 \sigma\left(F^{2}\right)\right]=0.062$

$w R\left(F^{2}\right)=0.168$

$S=1.02$

2140 reflections

$Z=1$

Mo $K \alpha$ radiation

$\mu=0.09 \mathrm{~mm}^{-1}$

$T=200 \mathrm{~K}$

$0.26 \times 0.23 \times 0.23 \mathrm{~mm}$

3505 measured reflections 2140 independent reflections 1258 reflections with $I>2 \sigma(I)$ $R_{\text {int }}=0.031$

Table 1

Hydrogen-bond geometry $\left(\AA{ }^{\circ}\right)$.

\begin{tabular}{lllll}
\hline$D-\mathrm{H} \cdots A$ & $D-\mathrm{H}$ & $\mathrm{H} \cdots A$ & $D \cdots A$ & $D-\mathrm{H} \cdots A$ \\
\hline $\mathrm{O} 1-\mathrm{H} 1 \cdots \mathrm{N} 1$ & 0.84 & 1.83 & $2.570(3)$ & 147 \\
\hline
\end{tabular}

Data collection: SMART (Bruker, 2000); cell refinement: SAINT (Bruker, 2000); data reduction: $S A I N T$; $\operatorname{program}(\mathrm{s})$ used to solve structure: SHELXS97 (Sheldrick, 2008); program(s) used to refine structure: SHELXL97 (Sheldrick, 2008); molecular graphics: ORTEP-3 (Farrugia, 1997) and PLATON (Spek, 2009); software used to prepare material for publication: SHELXL97.

This work was supported by Priority Research Centers Program through the National Research Foundation of Korea (NRF) funded by the Ministry of Education, Science and Technology (2010-0029626).

Supplementary data and figures for this paper are available from the IUCr electronic archives (Reference: DS2098).

\section{References}

Bermejo, M. R., Fernández, M. I., Gómez-Fórneas, E., González-Noya, A., Maneiro, M., Pedrido, R. \& Rodríguez, M. J. (2007). Eur. J. Inorg. Chem. pp. 3789-3797.

Bruker (2000). SADABS, SMART and SAINT. Bruker AXS Inc., Madison, Wisconsin, USA.

Farrugia, L. J. (1997). J. Appl. Cryst. 30, 565.

Fun, H.-K., Kia, R., Kargar, H. \& Jamshidvand, A. (2009). Acta Cryst. E65, o706.

Ha, K. (2010). Z. Kristallogr. New Cryst. Struct. 225, 737-738.

Sheldrick, G. M. (2008). Acta Cryst. A64, 112-122.

Spek, A. L. (2009). Acta Cryst. D65, 148-155.
138 parameters

$\Delta \rho_{\max }=0.23 \mathrm{e}^{-3}$

$\Delta \rho_{\min }=-0.21$ e $\AA^{-3}$ 


\section{supporting information}

Acta Cryst. (2011). E67, o918 [doi:10.1107/S1600536811009445]

\section{6,6'-Diethoxy-2,2'-[hexane-1,6-diylbis(nitrilomethanylylidene)]diphenol}

\section{Kwang Ha}

\section{S1. Comment}

The title compound crystallized in the triclinic space group $P \overline{1}$, same to the analogous compounds with propylene chain $\left(\mathrm{C}_{21} \mathrm{H}_{26} \mathrm{~N}_{2} \mathrm{O}_{4}\right)(\mathrm{Ha}, 2010)$ and butylene chain $\left(\mathrm{C}_{22} \mathrm{H}_{28} \mathrm{~N}_{2} \mathrm{O}_{4}\right)$ (Fun et al., 2009), whereas the related Schiff base with ethylene group $\left(\mathrm{C}_{20} \mathrm{H}_{24} \mathrm{~N}_{2} \mathrm{O}_{4}\right)$ crystallized in the monoclinic space group C2/c (Bermejo et al., 2007).

The asymmetric unit of the title molecule contains one half of the formula unit; a centre of inversion is located in the midpoint of the compound (Fig. 1). The Schiff base reveals strong intramolecular $\mathrm{O}-\mathrm{H} \cdots \mathrm{N}$ hydrogen bonding between the hydroxy $\mathrm{O}$ atom and the imino $\mathrm{N}$ atom with $\mathrm{d}(\mathrm{O} \cdots \mathrm{N})=2.570$ (3) $\AA$ forming a nearly planar six-membered ring (Fig. 2, Table 1). The $\mathrm{N} 1-\mathrm{C} 9 / 10$ bond lengths and the $\mathrm{C} 9-\mathrm{N} 1-\mathrm{C} 10$ bond angle indicate that the imino $\mathrm{N} 1$ atom is $s p^{2}$ hybridized $\left[d(\mathrm{~N} 1=\mathrm{C} 9)=1.271(3) \AA\right.$ and $\left.\mathrm{d}(\mathrm{N} 1-\mathrm{C} 10)=1.469(3) \AA ;<\mathrm{C} 9-\mathrm{N} 1-\mathrm{C} 10=121.2(2)^{\circ}\right]$. The torsion angles for the four atoms within the diiminohexylene chain indicate that the chain is almost perfectly in the anti conformation with $<\mathrm{N} 1-\mathrm{C} 10-\mathrm{C} 11-\mathrm{C} 12=179.1(2)^{\circ}$ and $<\mathrm{C} 10-\mathrm{C} 11-\mathrm{C} 12-\mathrm{C} 12^{\mathrm{i}}($ symmetry code $\mathrm{i}: 1-x, 2-y, 1-z)=$ $178.8(3)^{\circ}$.

\section{S2. Experimental}

1,6-Diaminohexane $(0.8132 \mathrm{~g}, 6.998 \mathrm{mmol})$ and 3-ethoxysalicylaldehyde $(2.3265 \mathrm{~g}, 14.000 \mathrm{mmol})$ in EtOH (20 ml) were stirred for $5 \mathrm{~h}$ at room temperature. After addition of pentane $(30 \mathrm{ml})$ to the reaction mixture, the formed precipitate was separated by filtration, washed with ether, and dried at $50{ }^{\circ} \mathrm{C}$, to give a yellow powder $(2.3563 \mathrm{~g})$. Crystals suitable for Xray analysis were obtained by slow evaporation from a toluene solution.

\section{S3. Refinement}

$\mathrm{H}$ atoms were positioned geometrically and allowed to ride on their respective parent atoms $[\mathrm{C}-\mathrm{H}=0.95 \AA(\mathrm{CH}), 0.99$ $\AA\left(\mathrm{CH}_{2}\right)$ or $0.98 \AA\left(\mathrm{CH}_{3}\right)$ and $\mathrm{O}-\mathrm{H}=0.84 \AA$, and $U_{\text {iso }}(\mathrm{H})=1.2 U_{\text {eq }}(\mathrm{C})$ or $1.5 U_{\text {eq }}($ methyl $\left.\mathrm{C}, \mathrm{O})\right]$. 


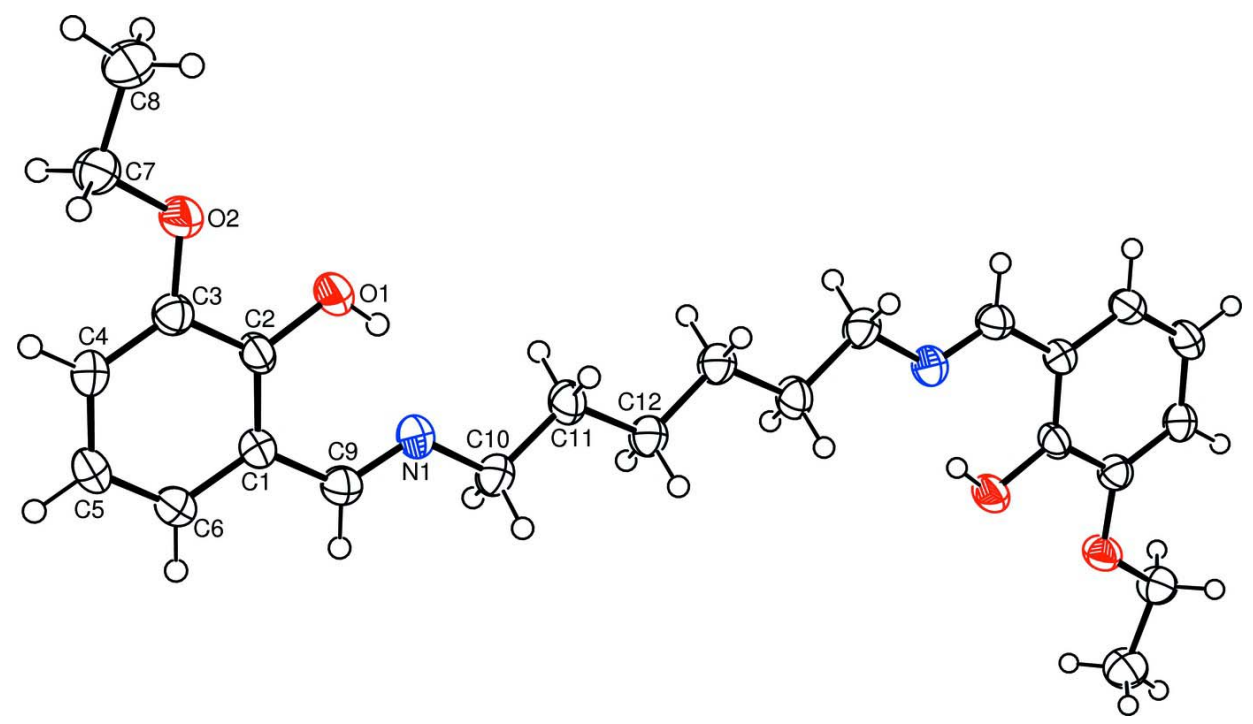

\section{Figure 1}

The structure of the title compound, with displacement ellipsoids drawn at the $50 \%$ probability level; $\mathrm{H}$ atoms are shown as small circles of arbitrary radius. Unlabelled atoms are related to the reference atoms by the $(1-x, 2-y, 1-z)$ symmetry transformation.

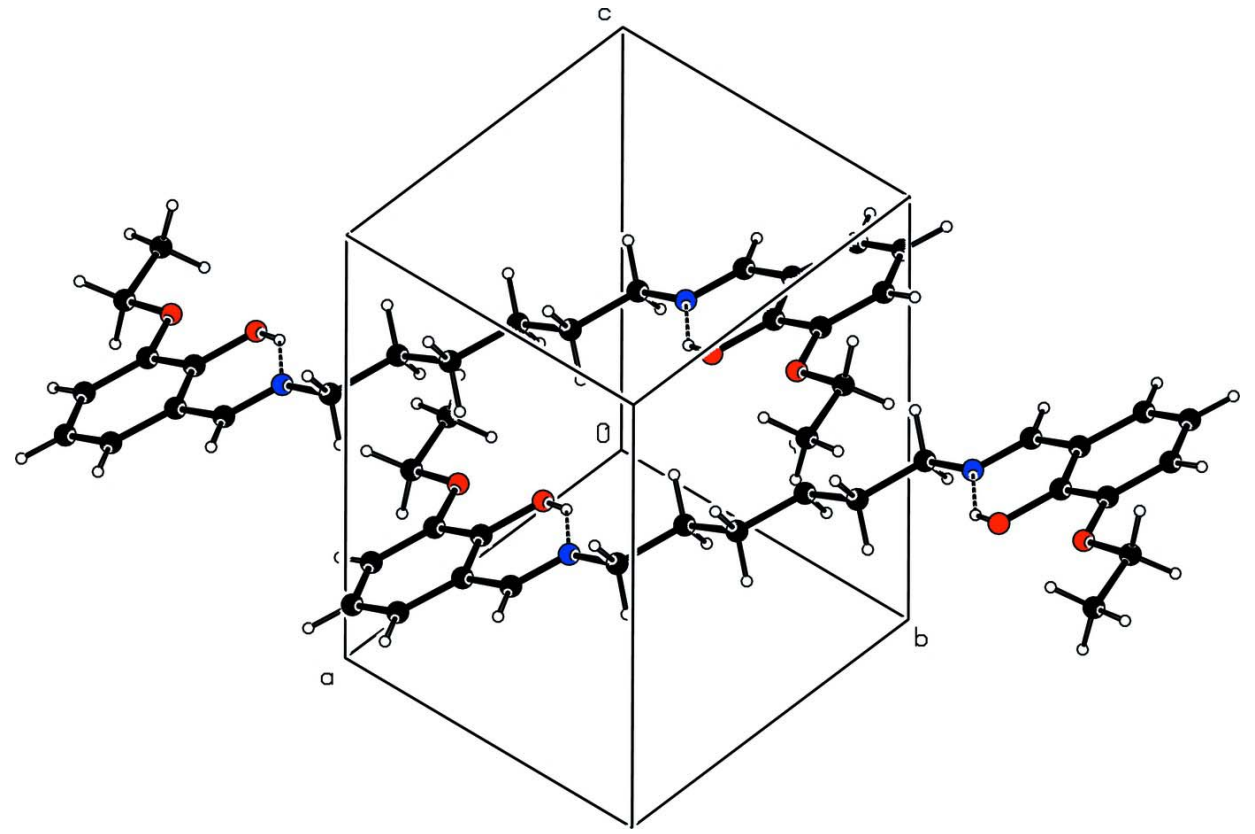

\section{Figure 2}

View of the unit-cell contents of the title compound. Hydrogen-bond interactions are drawn with dashed lines.

\section{2-ethoxy-6-\{[(6-\{[(3-ethoxy-2- hydroxyphenyl)methylidene]amino\}hexyl)imino]methyl\}phenol}

Crystal data

$\mathrm{C}_{24} \mathrm{H}_{32} \mathrm{~N}_{2} \mathrm{O}_{4}$

$M_{r}=412.52$

Triclinic, $P \overline{1}$
Hall symbol: -P 1

$a=6.9094(13) \AA$

$b=6.9184(13) \AA$ 
$c=11.936(2) \AA$

$\alpha=91.271(5)^{\circ}$

$\beta=99.677(4)^{\circ}$

$\gamma=102.550(4)^{\circ}$

$V=547.97(18) \AA^{3}$

$Z=1$

$F(000)=222$

$D_{\mathrm{x}}=1.250 \mathrm{Mg} \mathrm{m}^{-3}$

Data collection

Bruker SMART 1000 CCD

diffractometer

Radiation source: fine-focus sealed tube

Graphite monochromator

$\varphi$ and $\omega$ scans

Absorption correction: multi-scan

(SADABS; Bruker, 2000)

$T_{\min }=0.862, T_{\max }=1.000$

\section{Refinement}

Refinement on $F^{2}$

Least-squares matrix: full

$R\left[F^{2}>2 \sigma\left(F^{2}\right)\right]=0.062$

$w R\left(F^{2}\right)=0.168$

$S=1.02$

2140 reflections

138 parameters

0 restraints

Primary atom site location: structure-invariant direct methods
Mo $K \alpha$ radiation, $\lambda=0.71073 \AA$

Cell parameters from 873 reflections

$\theta=3.1-25.7^{\circ}$

$\mu=0.09 \mathrm{~mm}^{-1}$

$T=200 \mathrm{~K}$

Stick, yellow

$0.26 \times 0.23 \times 0.23 \mathrm{~mm}$

3505 measured reflections

2140 independent reflections

1258 reflections with $I>2 \sigma(I)$

$R_{\text {int }}=0.031$

$\theta_{\text {max }}=26.0^{\circ}, \theta_{\min }=3.0^{\circ}$

$h=-8 \rightarrow 8$

$k=-8 \rightarrow 8$

$l=-14 \rightarrow 11$

Secondary atom site location: difference Fourier map

Hydrogen site location: inferred from neighbouring sites

$\mathrm{H}$-atom parameters constrained

$w=1 /\left[\sigma^{2}\left(F_{\mathrm{o}}^{2}\right)+(0.0725 P)^{2}+0.0011 P\right]$

where $P=\left(F_{\mathrm{o}}^{2}+2 F_{\mathrm{c}}^{2}\right) / 3$

$(\Delta / \sigma)_{\max }<0.001$

$\Delta \rho_{\max }=0.23 \mathrm{e} \AA^{-3}$

$\Delta \rho_{\min }=-0.21 \mathrm{e} \AA^{-3}$

Special details

Geometry. All e.s.d.'s (except the e.s.d. in the dihedral angle between two 1.s. planes) are estimated using the full covariance matrix. The cell e.s.d.'s are taken into account individually in the estimation of e.s.d.'s in distances, angles and torsion angles; correlations between e.s.d.'s in cell parameters are only used when they are defined by crystal symmetry. An approximate (isotropic) treatment of cell e.s.d.'s is used for estimating e.s.d.'s involving 1.s. planes.

Refinement. Refinement of $F^{2}$ against ALL reflections. The weighted $R$-factor $w R$ and goodness of fit $S$ are based on $F^{2}$, conventional $R$-factors $R$ are based on $F$, with $F$ set to zero for negative $F^{2}$. The threshold expression of $F^{2}>\sigma\left(F^{2}\right)$ is used only for calculating $R$-factors(gt) etc. and is not relevant to the choice of reflections for refinement. $R$-factors based on $F^{2}$ are statistically about twice as large as those based on $F$, and $R$ - factors based on ALL data will be even larger.

Fractional atomic coordinates and isotropic or equivalent isotropic displacement parameters $\left(\hat{A}^{2}\right)$

\begin{tabular}{lllll}
\hline & $x$ & $y$ & $z$ & $U_{\text {iso }} * / U_{\text {eq }}$ \\
\hline O1 & $0.5421(2)$ & $0.2477(3)$ & $0.24546(17)$ & $0.0424(5)$ \\
$\mathrm{H} 1$ & 0.5714 & 0.3570 & 0.2834 & $0.064^{*}$ \\
$\mathrm{O} 2$ & $0.5003(2)$ & $-0.0878(2)$ & $0.13057(16)$ & $0.0405(5)$ \\
$\mathrm{N} 1$ & $0.7714(3)$ & $0.5589(3)$ & $0.35709(19)$ & $0.0383(6)$ \\
$\mathrm{C} 1$ & $0.9039(3)$ & $0.3153(3)$ & $0.2687(2)$ & $0.0312(6)$ \\
$\mathrm{C} 2$ & $0.7131(3)$ & $0.1965(3)$ & $0.2277(2)$ & $0.0318(6)$ \\
$\mathrm{C} 3$ & $0.6946(4)$ & $0.0168(4)$ & $0.1652(2)$ & $0.0327(6)$ \\
$\mathrm{C} 4$ & $0.8654(4)$ & $-0.0394(4)$ & $0.1439(2)$ & $0.0367(7)$ \\
$\mathrm{H} 4$ & 0.8526 & -0.1599 & 0.1007 & $0.044^{*}$
\end{tabular}




$\begin{array}{lllll}\text { C5 } & 1.0562(4) & 0.0782(4) & 0.1849(2) & 0.0399(7) \\ \text { H5 } & 1.1729 & 0.0374 & 0.1705 & 0.048^{*} \\ \text { C6 } & 1.0750(4) & 0.2543(4) & 0.2467(2) & 0.0357(7) \\ \text { H6 } & 1.2051 & 0.3348 & 0.2745 & 0.043^{*} \\ \text { C7 } & 0.4707(4) & -0.2781(3) & 0.0726(2) & 0.0400(7) \\ \text { H7A } & 0.5449 & -0.3639 & 0.1196 & 0.048^{*} \\ \text { H7B } & 0.5193 & -0.2641 & -0.0009 & 0.048^{*} \\ \text { C8 } & 0.2475(4) & -0.3664(4) & 0.0530(3) & 0.0492(8) \\ \text { H8A } & 0.2003 & -0.3725 & 0.1261 & 0.074^{*} \\ \text { H8B } & 0.2202 & -0.5005 & 0.0170 & 0.074^{*} \\ \text { H8C } & 0.1767 & -0.2835 & 0.0032 & 0.074^{*} \\ \text { C9 } & 0.9234(4) & 0.5017(4) & 0.3333(2) & 0.0351(6) \\ \text { H9 } & 1.0540 & 0.5831 & 0.3585 & 0.042^{*} \\ \text { C10 } & 0.7949(4) & 0.7477(4) & 0.4222(2) & 0.0394(7) \\ \text { H10A } & 0.8727 & 0.8568 & 0.3846 & 0.047^{*} \\ \text { H10B } & 0.8707 & 0.7421 & 0.4998 & 0.047^{*} \\ \text { C11 } & 0.5920(4) & 0.7884(3) & 0.4300(2) & 0.0384(7) \\ \text { H11A } & 0.5143 & 0.6766 & 0.4657 & 0.046^{*} \\ \text { H11B } & 0.5179 & 0.7939 & 0.3520 & 0.046^{*} \\ \text { C12 } & 0.6021(3) & 0.9794(4) & 0.4974(2) & 0.0366(7) \\ \text { H12A } & 0.6735 & 0.9729 & 0.5760 & 0.044^{*} \\ \text { H12B } & 0.6819 & 1.0911 & 0.4626 & 0.044^{*}\end{array}$

Atomic displacement parameters $\left(\AA^{2}\right)$

\begin{tabular}{lllllll}
\hline & $U^{11}$ & $U^{22}$ & $U^{33}$ & $U^{12}$ & $U^{13}$ & $U^{23}$ \\
\hline O1 & $0.0309(10)$ & $0.0439(11)$ & $0.0537(13)$ & $0.0112(8)$ & $0.0094(9)$ & $-0.0110(9)$ \\
O2 & $0.0304(10)$ & $0.0369(10)$ & $0.0523(13)$ & $0.0049(7)$ & $0.0070(9)$ & $-0.0091(9)$ \\
N1 & $0.0373(12)$ & $0.0389(13)$ & $0.0416(14)$ & $0.0150(10)$ & $0.0080(11)$ & $-0.0040(11)$ \\
C1 & $0.0289(13)$ & $0.0342(14)$ & $0.0323(15)$ & $0.0099(10)$ & $0.0068(12)$ & $0.0001(12)$ \\
C2 & $0.0278(13)$ & $0.0360(14)$ & $0.0356(16)$ & $0.0128(11)$ & $0.0096(12)$ & $0.0012(12)$ \\
C3 & $0.0330(14)$ & $0.0323(14)$ & $0.0331(15)$ & $0.0076(11)$ & $0.0061(12)$ & $0.0017(12)$ \\
C4 & $0.0387(14)$ & $0.0367(14)$ & $0.0372(16)$ & $0.0129(11)$ & $0.0087(13)$ & $-0.0053(12)$ \\
C5 & $0.0318(14)$ & $0.0461(16)$ & $0.0465(18)$ & $0.0148(12)$ & $0.0127(13)$ & $-0.0029(14)$ \\
C6 & $0.0282(13)$ & $0.0389(15)$ & $0.0401(16)$ & $0.0075(11)$ & $0.0066(12)$ & $-0.0007(12)$ \\
C7 & $0.0397(15)$ & $0.0322(15)$ & $0.0469(18)$ & $0.0078(11)$ & $0.0052(13)$ & $-0.0025(13)$ \\
C8 & $0.0450(16)$ & $0.0375(16)$ & $0.060(2)$ & $-0.0003(12)$ & $0.0075(15)$ & $-0.0070(14)$ \\
C9 & $0.0305(13)$ & $0.0347(14)$ & $0.0387(16)$ & $0.0049(11)$ & $0.0056(12)$ & $-0.0011(12)$ \\
C10 & $0.0410(15)$ & $0.0387(15)$ & $0.0385(17)$ & $0.0123(12)$ & $0.0037(13)$ & $-0.0083(13)$ \\
C11 & $0.0401(15)$ & $0.0351(15)$ & $0.0429(17)$ & $0.0121(11)$ & $0.0112(13)$ & $-0.0035(13)$ \\
C12 & $0.0381(15)$ & $0.0336(15)$ & $0.0387(16)$ & $0.0107(11)$ & $0.0056(13)$ & $-0.0032(12)$ \\
& & & & & & \\
\hline
\end{tabular}

Geometric parameters $\left(A,{ }^{\circ}\right)$

\begin{tabular}{llll}
\hline $\mathrm{O} 1-\mathrm{C} 2$ & $1.353(3)$ & $\mathrm{C} 7-\mathrm{C} 8$ & $1.506(4)$ \\
$\mathrm{O} 1-\mathrm{H} 1$ & 0.8400 & $\mathrm{C} 7-\mathrm{H} 7 \mathrm{~A}$ & 0.9900 \\
$\mathrm{O} 2-\mathrm{C} 3$ & $1.369(3)$ & $\mathrm{C} 7-\mathrm{H} 7 \mathrm{~B}$ & 0.9900 \\
$\mathrm{O} 2-\mathrm{C} 7$ & $1.431(3)$ & $\mathrm{C} 8-\mathrm{H} 8 \mathrm{~A}$ & 0.9800
\end{tabular}




\begin{tabular}{|c|c|c|c|}
\hline $\mathrm{N} 1-\mathrm{C} 9$ & $1.271(3)$ & $\mathrm{C} 8-\mathrm{H} 8 \mathrm{~B}$ & 0.9800 \\
\hline $\mathrm{N} 1-\mathrm{C} 10$ & $1.469(3)$ & $\mathrm{C} 8-\mathrm{H} 8 \mathrm{C}$ & 0.9800 \\
\hline $\mathrm{C} 1-\mathrm{C} 2$ & $1.396(3)$ & $\mathrm{C} 9-\mathrm{H} 9$ & 0.9500 \\
\hline $\mathrm{C} 1-\mathrm{C} 6$ & $1.401(3)$ & $\mathrm{C} 10-\mathrm{C} 11$ & $1.506(3)$ \\
\hline $\mathrm{C} 1-\mathrm{C} 9$ & $1.456(3)$ & $\mathrm{C} 10-\mathrm{H} 10 \mathrm{~A}$ & 0.9900 \\
\hline $\mathrm{C} 2-\mathrm{C} 3$ & $1.406(3)$ & $\mathrm{C} 10-\mathrm{H} 10 \mathrm{~B}$ & 0.9900 \\
\hline $\mathrm{C} 3-\mathrm{C} 4$ & $1.379(3)$ & $\mathrm{C} 11-\mathrm{C} 12$ & $1.514(3)$ \\
\hline $\mathrm{C} 4-\mathrm{C} 5$ & $1.393(3)$ & $\mathrm{C} 11-\mathrm{H} 11 \mathrm{~A}$ & 0.9900 \\
\hline $\mathrm{C} 4-\mathrm{H} 4$ & 0.9500 & $\mathrm{C} 11-\mathrm{H} 11 \mathrm{~B}$ & 0.9900 \\
\hline $\mathrm{C} 5-\mathrm{C} 6$ & $1.380(3)$ & $\mathrm{C} 12-\mathrm{C} 12^{\mathrm{i}}$ & $1.510(4)$ \\
\hline $\mathrm{C} 5-\mathrm{H} 5$ & 0.9500 & $\mathrm{C} 12-\mathrm{H} 12 \mathrm{~A}$ & 0.9900 \\
\hline $\mathrm{C} 6-\mathrm{H} 6$ & 0.9500 & $\mathrm{C} 12-\mathrm{H} 12 \mathrm{~B}$ & 0.9900 \\
\hline $\mathrm{C} 2-\mathrm{O} 1-\mathrm{H} 1$ & 109.5 & $\mathrm{C} 7-\mathrm{C} 8-\mathrm{H} 8 \mathrm{~A}$ & 109.5 \\
\hline $\mathrm{C} 3-\mathrm{O} 2-\mathrm{C} 7$ & $117.46(18)$ & $\mathrm{C} 7-\mathrm{C} 8-\mathrm{H} 8 \mathrm{~B}$ & 109.5 \\
\hline $\mathrm{C} 9-\mathrm{N} 1-\mathrm{C} 10$ & $121.2(2)$ & $\mathrm{H} 8 \mathrm{~A}-\mathrm{C} 8-\mathrm{H} 8 \mathrm{~B}$ & 109.5 \\
\hline $\mathrm{C} 2-\mathrm{C} 1-\mathrm{C} 6$ & $119.5(2)$ & $\mathrm{C} 7-\mathrm{C} 8-\mathrm{H} 8 \mathrm{C}$ & 109.5 \\
\hline $\mathrm{C} 2-\mathrm{C} 1-\mathrm{C} 9$ & $119.9(2)$ & $\mathrm{H} 8 \mathrm{~A}-\mathrm{C} 8-\mathrm{H} 8 \mathrm{C}$ & 109.5 \\
\hline $\mathrm{C} 6-\mathrm{C} 1-\mathrm{C} 9$ & $120.6(2)$ & $\mathrm{H} 8 \mathrm{~B}-\mathrm{C} 8-\mathrm{H} 8 \mathrm{C}$ & 109.5 \\
\hline $\mathrm{O} 1-\mathrm{C} 2-\mathrm{C} 1$ & $122.4(2)$ & $\mathrm{N} 1-\mathrm{C} 9-\mathrm{C} 1$ & $122.2(2)$ \\
\hline $\mathrm{O} 1-\mathrm{C} 2-\mathrm{C} 3$ & $117.9(2)$ & $\mathrm{N} 1-\mathrm{C} 9-\mathrm{H} 9$ & 118.9 \\
\hline $\mathrm{C} 1-\mathrm{C} 2-\mathrm{C} 3$ & $119.8(2)$ & $\mathrm{C} 1-\mathrm{C} 9-\mathrm{H} 9$ & 118.9 \\
\hline $\mathrm{O} 2-\mathrm{C} 3-\mathrm{C} 4$ & $126.0(2)$ & $\mathrm{N} 1-\mathrm{C} 10-\mathrm{C} 11$ & $110.5(2)$ \\
\hline $\mathrm{O} 2-\mathrm{C} 3-\mathrm{C} 2$ & $114.4(2)$ & $\mathrm{N} 1-\mathrm{C} 10-\mathrm{H} 10 \mathrm{~A}$ & 109.6 \\
\hline $\mathrm{C} 4-\mathrm{C} 3-\mathrm{C} 2$ & $119.6(2)$ & $\mathrm{C} 11-\mathrm{C} 10-\mathrm{H} 10 \mathrm{~A}$ & 109.6 \\
\hline $\mathrm{C} 3-\mathrm{C} 4-\mathrm{C} 5$ & $120.9(2)$ & $\mathrm{N} 1-\mathrm{C} 10-\mathrm{H} 10 \mathrm{~B}$ & 109.6 \\
\hline $\mathrm{C} 3-\mathrm{C} 4-\mathrm{H} 4$ & 119.6 & $\mathrm{C} 11-\mathrm{C} 10-\mathrm{H} 10 \mathrm{~B}$ & 109.6 \\
\hline $\mathrm{C} 5-\mathrm{C} 4-\mathrm{H} 4$ & 119.6 & $\mathrm{H} 10 \mathrm{~A}-\mathrm{C} 10-\mathrm{H} 10 \mathrm{~B}$ & 108.1 \\
\hline $\mathrm{C} 6-\mathrm{C} 5-\mathrm{C} 4$ & $119.7(2)$ & $\mathrm{C} 10-\mathrm{C} 11-\mathrm{C} 12$ & $114.0(2)$ \\
\hline $\mathrm{C} 6-\mathrm{C} 5-\mathrm{H} 5$ & 120.2 & $\mathrm{C} 10-\mathrm{C} 11-\mathrm{H} 11 \mathrm{~A}$ & 108.7 \\
\hline $\mathrm{C} 4-\mathrm{C} 5-\mathrm{H} 5$ & 120.2 & $\mathrm{C} 12-\mathrm{C} 11-\mathrm{H} 11 \mathrm{~A}$ & 108.7 \\
\hline $\mathrm{C} 5-\mathrm{C} 6-\mathrm{C} 1$ & $120.6(2)$ & $\mathrm{C} 10-\mathrm{C} 11-\mathrm{H} 11 \mathrm{~B}$ & 108.7 \\
\hline $\mathrm{C} 5-\mathrm{C} 6-\mathrm{H} 6$ & 119.7 & $\mathrm{C} 12-\mathrm{C} 11-\mathrm{H} 11 \mathrm{~B}$ & 108.7 \\
\hline $\mathrm{C} 1-\mathrm{C} 6-\mathrm{H} 6$ & 119.7 & $\mathrm{H} 11 \mathrm{~A}-\mathrm{C} 11-\mathrm{H} 11 \mathrm{~B}$ & 107.6 \\
\hline $\mathrm{O} 2-\mathrm{C} 7-\mathrm{C} 8$ & $106.4(2)$ & $\mathrm{C} 12^{\mathrm{i}}-\mathrm{C} 12-\mathrm{C} 11$ & $113.5(3)$ \\
\hline $\mathrm{O} 2-\mathrm{C} 7-\mathrm{H} 7 \mathrm{~A}$ & 110.4 & $\mathrm{C} 12^{\mathrm{i}}-\mathrm{C} 12-\mathrm{H} 12 \mathrm{~A}$ & 108.9 \\
\hline $\mathrm{C} 8-\mathrm{C} 7-\mathrm{H} 7 \mathrm{~A}$ & 110.4 & $\mathrm{C} 11-\mathrm{C} 12-\mathrm{H} 12 \mathrm{~A}$ & 108.9 \\
\hline $\mathrm{O} 2-\mathrm{C} 7-\mathrm{H} 7 \mathrm{~B}$ & 110.4 & $\mathrm{C} 12^{\mathrm{i}}-\mathrm{C} 12-\mathrm{H} 12 \mathrm{~B}$ & 108.9 \\
\hline $\mathrm{C} 8-\mathrm{C} 7-\mathrm{H} 7 \mathrm{~B}$ & 110.4 & $\mathrm{C} 11-\mathrm{C} 12-\mathrm{H} 12 \mathrm{~B}$ & 108.9 \\
\hline $\mathrm{H} 7 \mathrm{~A}-\mathrm{C} 7-\mathrm{H} 7 \mathrm{~B}$ & 108.6 & $\mathrm{H} 12 \mathrm{~A}-\mathrm{C} 12-\mathrm{H} 12 \mathrm{~B}$ & 107.7 \\
\hline $\mathrm{C} 6-\mathrm{C} 1-\mathrm{C} 2-\mathrm{O} 1$ & $-179.6(2)$ & $\mathrm{C} 3-\mathrm{C} 4-\mathrm{C} 5-\mathrm{C} 6$ & $-0.7(4)$ \\
\hline $\mathrm{C} 9-\mathrm{C} 1-\mathrm{C} 2-\mathrm{O} 1$ & $0.1(4)$ & $\mathrm{C} 4-\mathrm{C} 5-\mathrm{C} 6-\mathrm{C} 1$ & $0.2(4)$ \\
\hline $\mathrm{C} 6-\mathrm{C} 1-\mathrm{C} 2-\mathrm{C} 3$ & $0.2(4)$ & $\mathrm{C} 2-\mathrm{C} 1-\mathrm{C} 6-\mathrm{C} 5$ & $0.0(4)$ \\
\hline $\mathrm{C} 9-\mathrm{C} 1-\mathrm{C} 2-\mathrm{C} 3$ & $179.9(2)$ & $\mathrm{C} 9-\mathrm{C} 1-\mathrm{C} 6-\mathrm{C} 5$ & $-179.6(3)$ \\
\hline $\mathrm{C} 7-\mathrm{O} 2-\mathrm{C} 3-\mathrm{C} 4$ & $3.8(4)$ & $\mathrm{C} 3-\mathrm{O} 2-\mathrm{C} 7-\mathrm{C} 8$ & $175.5(2)$ \\
\hline $\mathrm{C} 7-\mathrm{O} 2-\mathrm{C} 3-\mathrm{C} 2$ & $-176.2(2)$ & $\mathrm{C} 10-\mathrm{N} 1-\mathrm{C} 9-\mathrm{C} 1$ & $-179.9(3)$ \\
\hline $\mathrm{O} 1-\mathrm{C} 2-\mathrm{C} 3-\mathrm{O} 2$ & $-0.9(4)$ & $\mathrm{C} 2-\mathrm{C} 1-\mathrm{C} 9-\mathrm{N} 1$ & $2.1(4)$ \\
\hline
\end{tabular}




\section{supporting information}

$\begin{array}{llll}\mathrm{C} 1-\mathrm{C} 2-\mathrm{C} 3-\mathrm{O} 2 & 179.3(2) & \mathrm{C} 6-\mathrm{C} 1-\mathrm{C} 9-\mathrm{N} 1 & -178.3(3) \\ \mathrm{O} 1-\mathrm{C} 2-\mathrm{C} 3-\mathrm{C} 4 & 179.1(2) & \mathrm{C} 9-\mathrm{N} 1-\mathrm{C} 10-\mathrm{C} 11 & 175.2(3) \\ \mathrm{C} 1-\mathrm{C} 2-\mathrm{C} 3-\mathrm{C} 4 & -0.7(4) & \mathrm{N} 1-\mathrm{C} 10-\mathrm{C} 11-\mathrm{C} 12 & 179.1(2) \\ \mathrm{O} 2-\mathrm{C} 3-\mathrm{C} 4-\mathrm{C} 5 & -179.1(2) & \mathrm{C} 10-\mathrm{C} 11-\mathrm{C} 12-\mathrm{C} 12^{\mathrm{i}} & 178.8(3) \\ \mathrm{C} 2-\mathrm{C} 3-\mathrm{C} 4-\mathrm{C} 5 & 0.9(4) & & \end{array}$

Symmetry code: (i) $-x+1,-y+2,-z+1$.

Hydrogen-bond geometry $\left(\AA,{ }^{\circ}\right)$

\begin{tabular}{lllll}
\hline$D-\mathrm{H} \cdots A$ & $D-\mathrm{H}$ & $\mathrm{H} \cdots A$ & $D \cdots A$ & $D-\mathrm{H} \cdots A$ \\
\hline $\mathrm{O} 1-\mathrm{H} 1 \cdots \mathrm{N} 1$ & 0.84 & 1.83 & $2.570(3)$ & 147 \\
\hline
\end{tabular}

